\title{
Numerical Analyses Optical Solitons in Dual Core Couplers with Kerr Law Nonlinearity
}

\author{
A. A. Al Qarni1, M. A. Banaja2 ${ }^{2}$ H. O. Bakodah' ${ }^{2}$ \\ ${ }^{1}$ Department of Mathematics, Faculty of Science for Girls, Bisha University, Bisha, Saudi Arabia \\ ${ }^{2}$ Department of Mathematics, Faculty of Science for Girls, King Abdulaziz University, Jeddah, Saudi Arabia \\ Email: alyaa14092009@hotmail.com
}

Received 27 May 2015; accepted 6 November 2015; published 9 November 2015

Copyright (C) 2015 by authors and Scientific Research Publishing Inc.

This work is licensed under the Creative Commons Attribution International License (CC BY).

http://creativecommons.org/licenses/by/4.0/

(c) (i) Open Access

\begin{abstract}
In this paper, we present the results of numerical analysis of optical solitons in dual core couplers. We studied the optical couplers as an application for the non-linear Schrödinger equation in the case of Kerr law for non-linear and clarify the exact solution in this case. Then we have provided a numerical study of the effect of changing the constants in the form of the three types of solitons: bright soliton and dark solitons and singular soliton.
\end{abstract}

Keywords

Optical Soliton, Dual Core Couplers, Kerr Law Nonlinearity

\section{Introduction}

The propagation solitons through optical fibers have been a major area of study given its potential applicability in optical communication systems. Several effects that are present in optical fibers and amplifiers limit the performance of optical system. Signal propagation through optical fibers can be affected by group velocity dispersion (GVD), polarization mode dispersion (PMD), and nonlinear effects. The main nonlinear effects that arise in monomode fibers are Brillion scattering, Raman scattering, and the Kerr effect. The Kerr effect of nonlinearity is due to the dependence of the fiber refractive index on the field intensity. The intensity dependence of the refractive index leads to a larger number of interesting nonlinear effects. Notable among them, which have been studied widely, are self-phase modulation (SPM) and cross phase modulation (XPM). The propagation solitons through optical fibers have been well established that this dynamics is described, to first approximation, by the integral nonlinear Schrodinger equation (NLSE) [1]. The Nonlinear Schrodinger's Equation plays a vital role in various areas of physical, biological, and engineering sciences. It appears in many applied fields, including fluid dynamics, nonlinear optics, plasma physics, and protein chemistry. The NLSE that is going to be studied is giv- 
en by

$$
i q_{t}+\frac{1}{2} q_{x x}+F|q|^{2} q=(0)
$$

$F$ is a real-valued algebraic function, $q$ is the dependent variable, $x$ and $t$ are the independent variables.

Equation (1) is known to support solitons or soliton solutions for various kinds of nonlinearity.

There are various kinds of nonlinearities of the function $F$ in (1) that are known so far.

The special case where $F(s)=s$, also known as the kerr law of nonlinearity, is integrable by the method of inverse scattering transform (IST) [2]-[5].

The Kerr law of nonlinearity originates from the fact that a light wave in an optical fiber faces nonlinear responses from nonharmonic motion of electrons bound in molecules, caused by an external electric field. Even though the nonlinear responses are extremely weak, their effects appear in various ways over long distances of propagation that are measured in terms of light wavelength.

\section{Dual-Core Couplers}

Nonlinear couplers are very useful devices that distribute light from a main fiber into one or more branch fibers. Couplers also have applications as intensity dependent switches and as limiters.

Switching is the process of energy redistribution between the cores for a given input. The problem of switching, although involved, can be accomplished when the stability of soliton states is known.

Although there has been a lot of work in the area of optical couplers, our approach in this paper is going to present the special cases of the kerr-law nonlinearity.

Also, there has been a lot of exact and approximate numerical studies in the context of optical couplers. But these numerical techniques could get computationally intense [6] [7].

For Dual-core couplers, wave propagation at relatively high field intensities is described by coupled nonlinear equations. In the dimensionless form, they are

$$
\begin{aligned}
& i q_{t}+a_{1} q_{x x}+b_{1} F\left(|q|^{2}\right) q=K_{1} r \\
& i r_{t}+a_{2} r_{x x}+b_{2} F\left(|r|^{2}\right) r=K_{2} q
\end{aligned}
$$

Equations (2) and (3) represent a generic model to study the dynamics of optical solitons through dual-core optical couplers.

The first term in both equations represent linear temporal evolution. The coefficients of $a_{l}$ for $l=1,2$ is the group velocity dispersion (GVD) while $b_{l}$ represent non-Kerr law nonlinearity, in general. On the right hand sides $K_{l}$ are the coupling coefficients. The dependent variables $q(x, t)$ and $r(x, t)$ are the complex-valued wave profiles that propagate through these couplers. In this research, the focus is limited to soliton signals.

The functional $F$ represents non-Kerr law nonlinearity, in general.

For Kerr law nonlinearity, $F(s)=s$. The model Equations (2) and (3) reduces to

$$
\begin{aligned}
& i q_{t}+a q_{x x}+b_{1}|q|^{2} q=K_{1} r \\
& i r_{t}+a r_{x x}+b_{2}|r|^{2} r=K_{2} q
\end{aligned}
$$

For integrality aspects of this coupled equations by ansatz method an assumption of the following form is considered:

$$
\begin{aligned}
& q(x, t)=P_{1}(x, t) \mathrm{e}^{i \phi(x, t)} \\
& r(x, t)=P_{2}(x, t) \mathrm{e}^{i \phi(x, t)}
\end{aligned}
$$

where $P_{l}(x, t)(l=1 ; 2)$ represents the amplitude components of soliton while the phase component $\phi(x, t)$ is

$$
\phi(x, t)=-k x+\omega t+\theta
$$


In (6), $K$ is the frequency of the solution's while $\omega$ represents the wave number and $\theta$ is the phase constant. Substituting (6) and (7) into (4) and (5) and then decomposing into real and imaginary parts give

$$
a_{l} \frac{\partial^{2} P_{l}}{\partial x^{2}}-P_{l}\left(\omega+a_{l} k^{2}\right)+b_{l} F\left(\left|P_{l}\right|^{2}\right) P_{l}-K_{l} P_{i}=0
$$

and

$$
\frac{\partial P_{l}}{\partial t}-2 a_{l} k \frac{\partial P_{l}}{\partial x}=0
$$

Respectively.

From the imaginary part equation it is possible to obtain the speed $(v)$ of the soliton as,

$$
v=-2 a_{l} K
$$

Since $P_{l}(x, t)$ can be represented as $P_{l}(x-v t)$, where the function $g$ is the soliton wave profile depending the type of nonlinearity and $v$ is the speed of the soliton.

Now, equating the two values of the soliton speed, from (11) leads to

$$
a_{1}=a_{2}
$$

The speed of the soliton therefore reduces to

$$
v=-2 a K
$$

The coupled NLSE for dual-core couplers given by (4) and (5) modifies to

$$
\begin{aligned}
& i q_{t}+a_{1} q_{x x}+b_{1} F\left(|q|^{2}\right) q=K_{1} r \\
& i r_{t}+a_{2} r_{x x}+b_{2} F\left(|r|^{2}\right) r=K_{2} q
\end{aligned}
$$

where $a_{1}=a_{2}=a$ Consequently, the real part Equation (9) reduces to

$$
a \frac{\partial^{2} P_{l}}{\partial x^{2}}-P_{l}\left(\omega+a_{l} k^{2}\right)+b_{l} P_{l}^{3}-K_{l} P_{\grave{i}}=0
$$

This equation will now be integrated for three types of solitons. They are bright, dark and singular soliton solutions.

\section{Families of Soliton Solutions}

\subsection{Bright Solitons}

For bright solitons, one assumes [8] [9]

$$
P_{l}=A_{1} \operatorname{sech}^{P_{1}} \tau
$$

where;

$$
\tau=B(x-v t)
$$

Here, $A_{1}$ represents the soliton amplitude and $B$ is the inverse width of the soliton.

Substituting (17) into (16) gives:

$$
\begin{aligned}
& A_{l}\left(\omega+a k^{2}-a p_{l}^{2} B^{2}\right) \operatorname{sech}^{p_{l}} \tau+a A_{l} p_{l} B^{2}\left(p_{l}+1\right) \operatorname{sech}^{p_{l}+2} \\
& -b_{l}\left(A_{l}^{3} \operatorname{sech}^{3 p_{l}} \tau\right)+K_{l} A_{l} \operatorname{sech}^{P_{l}} \tau=0
\end{aligned}
$$

Balancing principle yields

$$
p_{l}+2=3 p_{l}
$$

So that

$$
p_{l}=1 \text { for } l=1,2 \text {. }
$$


Substituting (21) into (19) we get:

$$
A_{l}\left(\omega+a k^{2}-a B^{2}\right) \operatorname{sech} \tau+\left(2 a A_{1} B^{2}-b_{l} A_{l}^{3}\right) \operatorname{sech}^{3} \tau+K_{l} A_{l} \operatorname{sech} \tau=0
$$

From coefficient sech $\tau$ into (22) we get $\omega+a k^{2}-a B^{2}+K_{l} \frac{A_{l}}{A_{l}}=0$ and therefore:

$$
\omega=a\left(B^{2}-k^{2}\right)-K_{l} \frac{A_{1}}{A_{l}}
$$

From coefficient $\operatorname{sech}^{3} \tau$, we get $2 a A_{1}^{2} B^{2}-b=0$ and therefore:

$$
B=\sqrt{\frac{b_{1}}{2 a}} A_{1}
$$

which poses the constraint

$$
a b_{l}>0 \text { for } l=1,2 .
$$

This means that the GVD and Kerr law nonlinearity must bear the same sign for bright solitons to exist.

Next, equating the width of the solitons for $l=1 ; 2$ from (24) imply $b_{1} A_{1}^{2}=b_{2} A_{2}^{2}$ And therefore:

$$
\frac{A_{1}}{A_{2}}=\sqrt{\frac{b_{2}}{b_{1}}}
$$

which again shows the

$$
b_{1} b_{2}>0
$$

This shows that the nonlinearity of the two cores must also carry the same sign. Then, equating the wave numbers for the two components gives the relation (23) we get $K_{1} A_{2}^{2}=K_{2} A_{1}^{2}$ and therefore:

$$
\frac{A_{1}}{A_{2}}=\sqrt{\frac{K_{1}}{K_{2}}}
$$

which again shows that

$$
K_{1} K_{2}>0
$$

Finally equating (26) and (28) leads to

$$
b_{1} K_{1}=b_{2} K_{2}
$$

which is the constraint condition between the given coefficients that must hold for bright solitons to exist. This leads to the bright 1 -soliton solution for dual couplers:

$$
\begin{aligned}
& q(x, t)=A_{1} \operatorname{sech}[B(x-v t)] \mathrm{e}^{i(-k x+\omega t+\theta)} \\
& r(x, t)=A_{2} \operatorname{sech}[B(x ? v t)]^{i(-k x+\omega t+\theta)}
\end{aligned}
$$

which will exist for the necessary constraints in place.

\subsection{Dark Solitons}

For dark solitons, the starting hypothesis is given by [6]

$$
P_{l}=A_{l} \tanh ^{p_{l}} \tau
$$

with the definition of $\tau$ being the same in (18). However for dark solitons the parameters $A_{1}$ and $B$ are free parameters. Substituting (33) and (18) into (16) leads to

$$
\begin{aligned}
& A_{l}\left(\omega+a k^{2}+2 a p_{l}^{2} B^{2}\right) \tanh ^{p_{l}} \tau-a A_{l} B^{2} p_{l}\left(p_{l}-1\right) \tanh ^{P_{l}-2} \tau \\
& -a A_{l} p_{l} B^{2}\left(p_{l}+1\right) \tanh ^{p_{l}+2} \tau-b_{l} A_{l}^{3} \tanh ^{3 p_{l}} \tau+K_{l} A_{l} \tanh ^{p_{l}} \tau=0
\end{aligned}
$$


Balancing principle yields

$$
p_{l}+2=3 p_{l}
$$

So that

$$
p_{l}=1 \quad \text { for } l=1,2 \text {. }
$$

Substituting (36) into (34) we get:

$$
A_{l}\left(\omega+a k^{2}-a B^{2}\right) \tanh \tau+\left(2 a A_{l} B^{2}-b_{l} A_{l}^{3}\right) \tanh ^{3} \tau+K_{l} A_{l} \tanh \tau=0
$$

From coefficient $\tanh ^{1} \tau$ we get $\omega+a k^{2}-a B^{2}+\frac{A_{1}}{A_{1}}=0$ and therefore:

$$
\omega=a\left(B^{2}-k^{2}\right)-K_{l} \frac{A_{1}}{A_{l}}
$$

From coefficient $\tanh ^{3} \tau$ we get $2 a A_{1}^{2} B^{2}-b=0$ and therefore:

$$
B=\sqrt{\frac{b_{1}}{2 a}} A_{1}
$$

which poses the constraint

$$
a b_{l}>0 \text { for } l=1,2 .
$$

This means that the GVD and Kerr law nonlinearity must bear the same sign for bright solitons to exist.

Next, equating the width of the solitons for $l=1 ; 2$ from (39) implied $b_{1} A_{1}^{2}=b_{2} A_{2}^{2}$ and therefore:

$$
\frac{A_{1}}{A_{2}}=\sqrt{\frac{b_{2}}{b_{1}}}
$$

which again shows that

$$
b_{1} b_{2}>0
$$

This shows that the nonlinearity of the two cores must also carry the same sign. Then, equating the wave numbers for the two components gives the relation (38) we get $K_{1} A_{2}^{2}=K_{2} A_{1}^{2}$ And therefore:

$$
\frac{A_{1}}{A_{2}}=\sqrt{\frac{K_{1}}{K_{2}}}
$$

which again shows that

$$
K_{1} K_{2}>0
$$

Finally equating (41) and (43) leads to

$$
b_{1} K_{1}=b_{2} K_{2}
$$

This gives dark 1-soliton solution for dual-core couplers

$$
\begin{aligned}
& q(x, t)=A_{1} \tanh [B(x-v t)] \mathrm{e}^{i(-k x+\omega t+\theta)} \\
& r(x, t)=A_{2} \tanh [B(x-v t)] \mathrm{e}^{i(-k x+\omega t+\theta)}
\end{aligned}
$$

along with their respective constraints as indicated.

Note: These waves known as check waves.

\subsection{Singular Solitons}

For singular solitons, the starting hypothesis is given by [6] 


$$
P_{l}=A_{1} \operatorname{csch}^{P_{1}} \tau
$$

where $\tau$ is the same as in (18) while the parameters $A_{1}$ and $B$ are again free parameters. Upon substituting (48) and (16) into (18) gives

$$
A_{l}\left(\omega+a k^{2}-a p_{l}^{2} B^{2}\right)-a A_{l} B^{2} p_{l}\left(p_{l}+1\right) \operatorname{csch}^{p_{l}+2} \tau-b_{l} A_{l}^{3} \operatorname{csch}^{3 p_{l}} \tau+K_{l} A_{l} \operatorname{csch}^{p_{l}} \tau=0
$$

Balancing principle yields

$$
p_{l}+2=3 p_{l}
$$

So that

$$
p_{l}=1 \quad \text { for } l=1,2 \text {. }
$$

Substituting (51) into (49) we get:

$$
A_{l}\left(\omega+a k^{2}-a B^{2}\right) \operatorname{csch} \tau+\left(2 a A_{1} B^{2}-b_{l} A_{l}^{3}\right) \operatorname{csch}^{3} \tau+K_{l} A_{l} \operatorname{csch} \tau=0
$$

From coefficient $\operatorname{csch} \tau$ we get $\omega+a k^{2}-a B^{2}+K_{l} \frac{A_{l}}{A_{l}}=0$ and therefore:

$$
\omega=a\left(B^{2}-k^{2}\right)-K_{l} \frac{A_{l}}{A_{l}}
$$

From coefficient $\operatorname{csch}^{3} \tau$ we get $2 a A_{1}^{2} B^{2}-b=0$ and therefore:

$$
B=\sqrt{\frac{b_{l}}{2 a}} A_{1}
$$

which poses the constraint

$$
a b_{l}>0 \text { for } l=1,2 \text {. }
$$

This means that the GVD and Kerr law nonlinearity must bear the same sign for bright solitons to exist.

Next, equating the width of the solitons for $l=1 ; 2$ from (54) implied $b_{1} A_{1}^{2}=b_{2} A_{2}^{2}$. And therefore:

$$
\frac{A_{1}}{A_{2}}=\sqrt{\frac{b_{2}}{b_{1}}}
$$

which again shows the

$$
b_{1} b_{2}>0
$$

This shows that the nonlinearity of the two cores must also carry the same sign. Then, equating the wave numbers for the two components gives the relation (53) we get $K_{1} A_{2}^{2}=K_{2} A_{1}^{2}$. And therefore:

$$
\frac{A_{1}}{A_{2}}=\sqrt{\frac{K_{1}}{K_{2}}}
$$

which again shows that

$$
K_{1} K_{2}>0
$$

Finally equating (56) and (58) leads to

$$
b_{1} K_{1}=b_{2} K_{2}
$$

These lead to singular 1-soliton solutions in dual-core optical fibers with Kerr nonlinearity given by

$$
\begin{aligned}
& q(x, t)=A_{1} \operatorname{csch}[B(x-v t)] \mathrm{e}^{i(-K x+\omega t+\theta)} \\
& r(x, t)=A_{2} \operatorname{csch}[B(x-v t)] \mathrm{e}^{i(-K x+\omega t+\theta)}
\end{aligned}
$$

which will exist for the necessary constraints in place. 


\section{Results of Numerical Analyses}

Analytic process of the CNLS can only be found under certain special selections of parameters, i.e. certain underlying physical system. For other cases, numerical analysis is necessary for seeking the evolution of solutions. It is essential to select a suitable numerical method for solving the equations [10].

To study the effect of parameters on Solitons we consider the following values [11]:

Case 1: if they

$$
\begin{gathered}
b_{1}=\frac{1}{2}, K_{2}=\frac{6}{b_{2}}, A_{1}=1.0103, A_{2}=\frac{A_{1}}{\sqrt{b_{2}}}, v=-2 a k, \\
a=\frac{1}{2}, k=1, K_{1}=6, \omega=3, B=\sqrt{\frac{b_{2}}{2 a}} A_{2}, \quad \theta=\pi
\end{gathered}
$$

Studying the effect of changing $b_{2}$ (non-linear coefficient) in terms of $b_{1}$ so take three cases

$$
b_{2}=\frac{1}{4} b_{1}, b_{2}=b_{1}, b_{2}=10 b_{1}
$$

Figures 1-3 shows the effect of this change on Soliton amplitude.

Case 2: if they

$$
\begin{aligned}
& b_{1}=\frac{1}{2}, K_{2}=\frac{6}{b_{2}}, A_{1}=1.0103, A_{2}=\frac{A_{1}}{\sqrt{b_{2}}}, v=-2 a k, \\
& b_{2}=\frac{1}{4} b_{1}, k=1, K_{1}=6, \omega=3, \quad B=\sqrt{\frac{b_{2}}{2 a}} A_{2}, \quad \theta=\pi
\end{aligned}
$$

Studying the effect of changing a (velocity dispersion coefficient) on the wave form and the values

$$
a=\frac{1}{2}, a=1, a=2
$$

Figure 4-6 shows the effect of this change on the width Soliton.

Case 3: if they

$$
\begin{gathered}
b_{1}=\frac{1}{2}, K_{2}=\frac{6}{b_{2}}, a=\frac{1}{2}, A_{2}=\frac{A_{1}}{\sqrt{b_{2}}}, v=-2 a k, \\
b_{2}=\frac{1}{4} b_{1}, k=1, K_{1}=6, \omega=3, \quad B=\sqrt{\frac{b_{2}}{2 a}} A_{2}, \quad \theta=\pi
\end{gathered}
$$

Studying the effect of changing $A_{1}$ (Soliton amplitude) on the wave form, and that the values of

$$
A_{1}=0.550515, A_{1}=1.0103, A_{1}=2.20206
$$

Figures 7-9 shows the effect of this change on the Soliton width and Soliton amplitude.

\section{Conclusions}

The study couplers dual core is considered one of modern topics of great importance in the field of optical communication. In this research, the Schrodinger equation is linear been studying these couplers in the case of kerr law nonlinearity and clarifying the exact solution in this case.

And we studied the effect of changing constants derived under the restrictions mentioned on Soliton form in the case of Solitons bright as in the case of a change $b_{2}$ (non-linear coefficient) impact on soliton amplitude. When you changed $a$ (velocity dispersion coefficient), we found that the effect on Soliton width as well as when changing $A_{1}$ (Soliton amplitude), the impact on the Soliton width and Soliton amplitude.

Similarly, these changes have in the case of dark Solitons and singular Solitons.

The extension of this work can apply different types of non-linear as well as increase the Perturbation terms to 

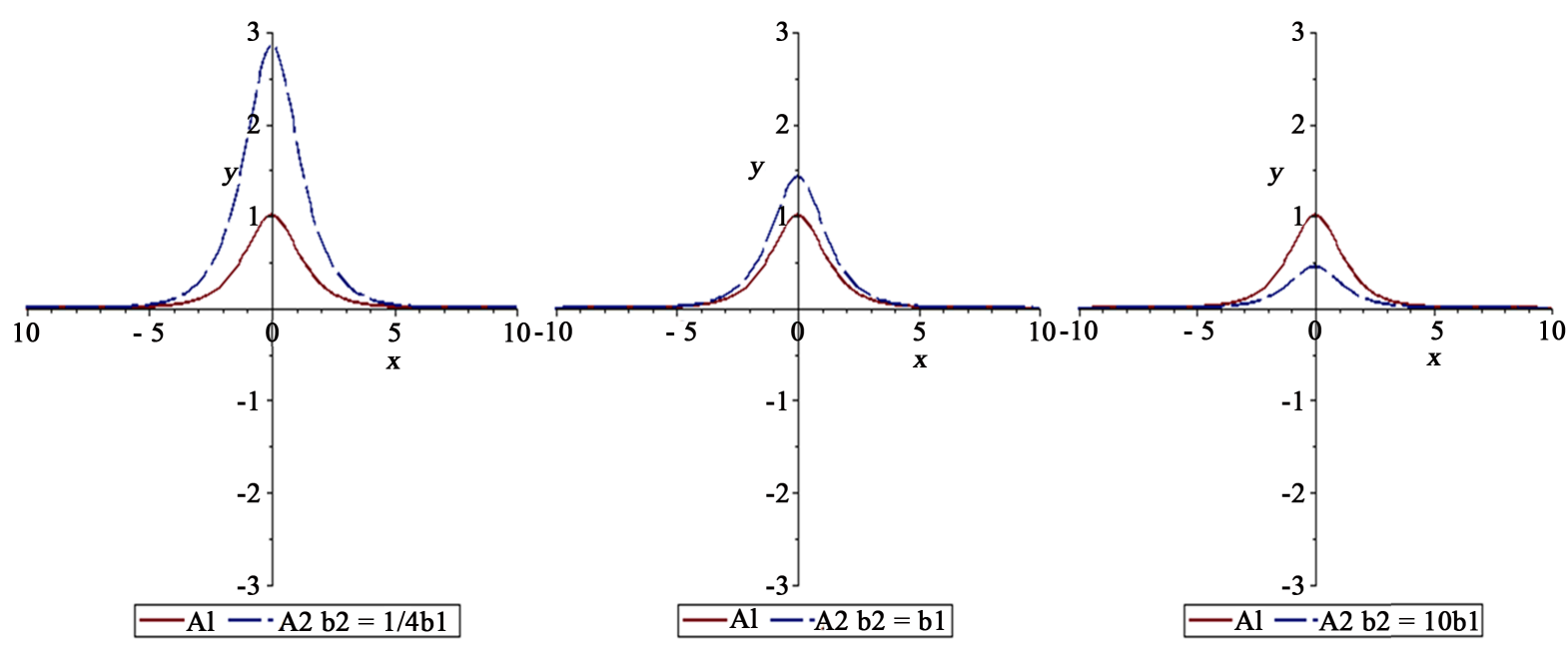

Figure 1. Bright solitons.
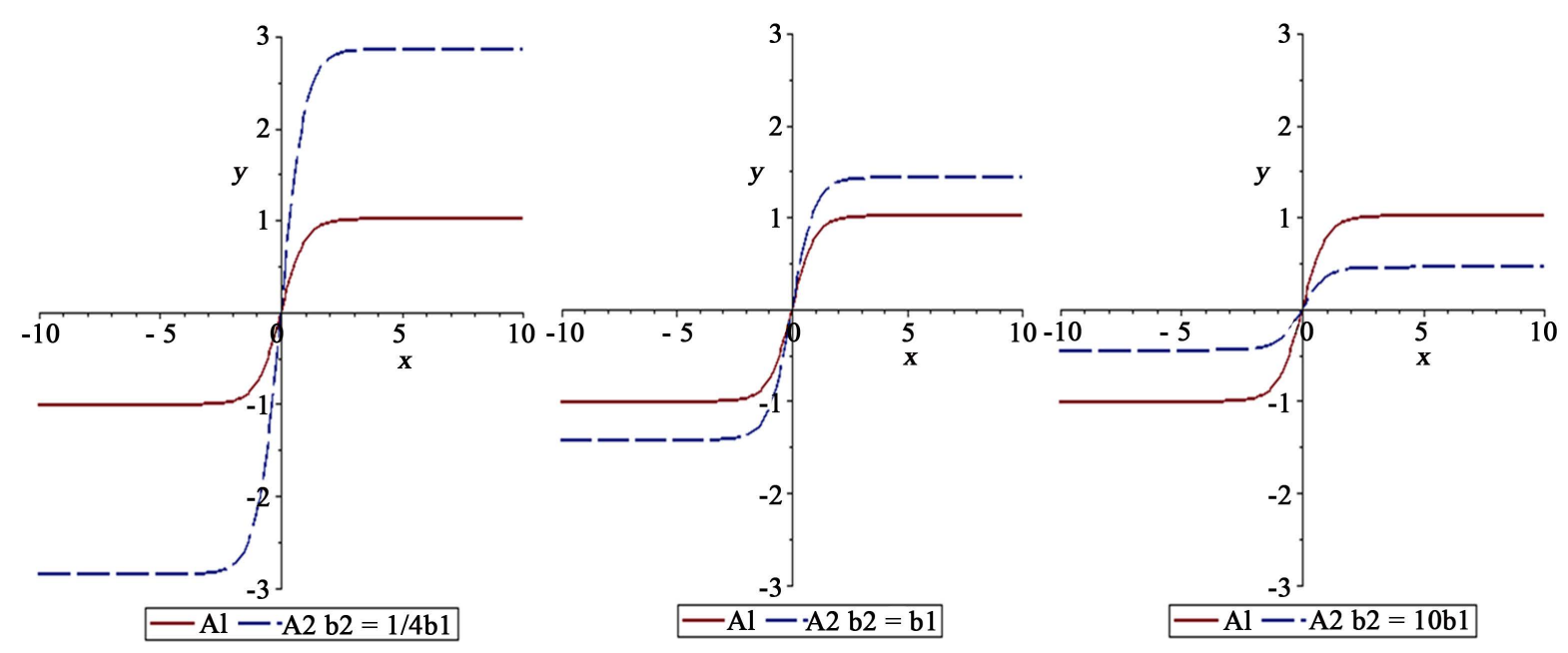

Figure 2. Dark solitons.
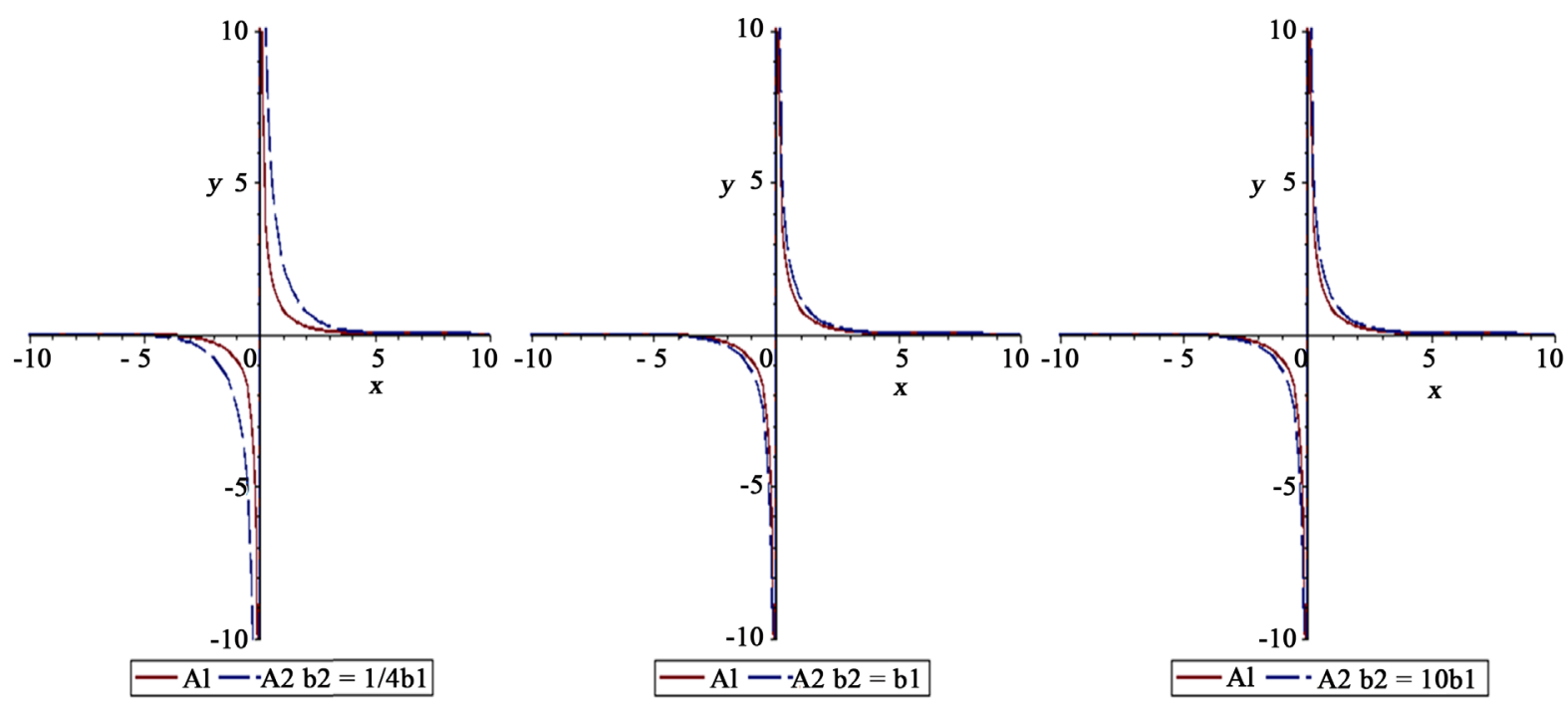

Figure 3. Singular solitons. 
A. A. Al Qarni et al.

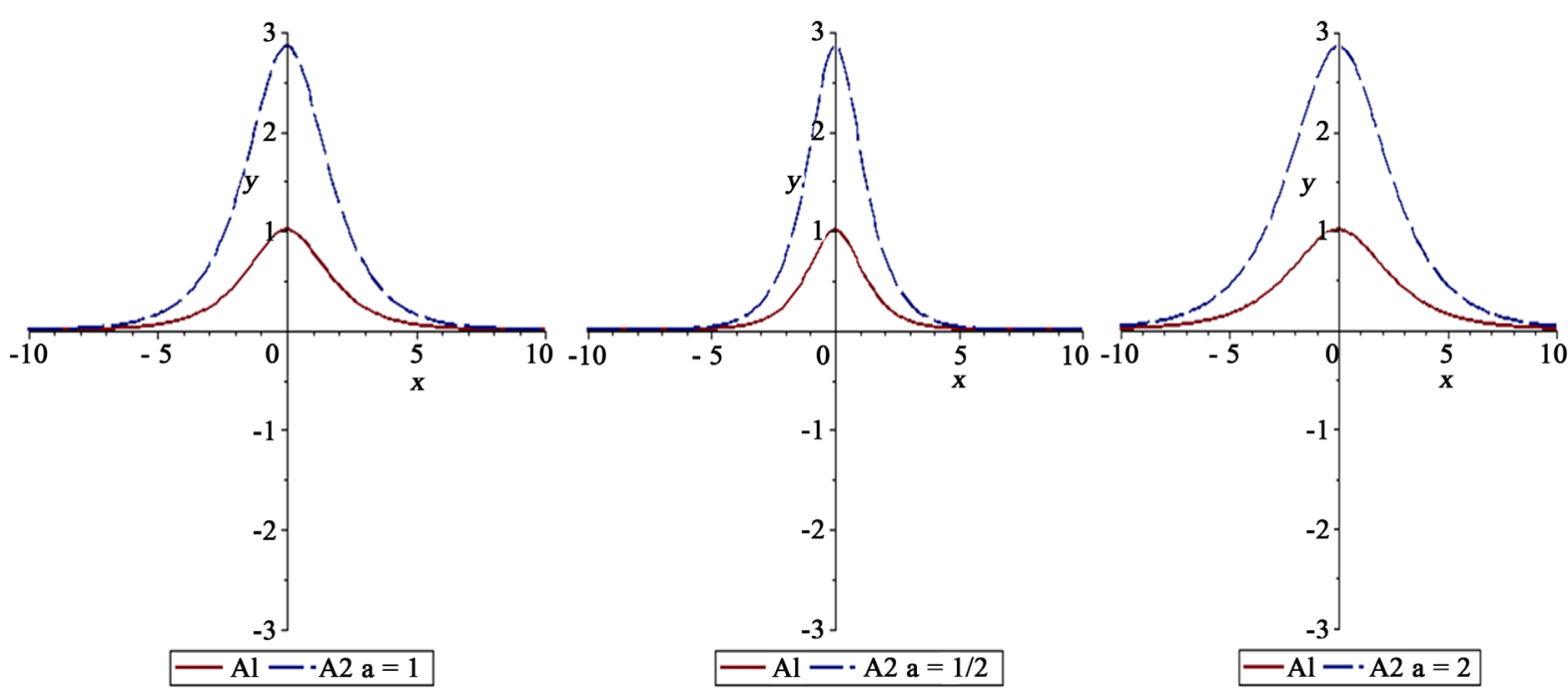

Figure 4. Bright solitons.
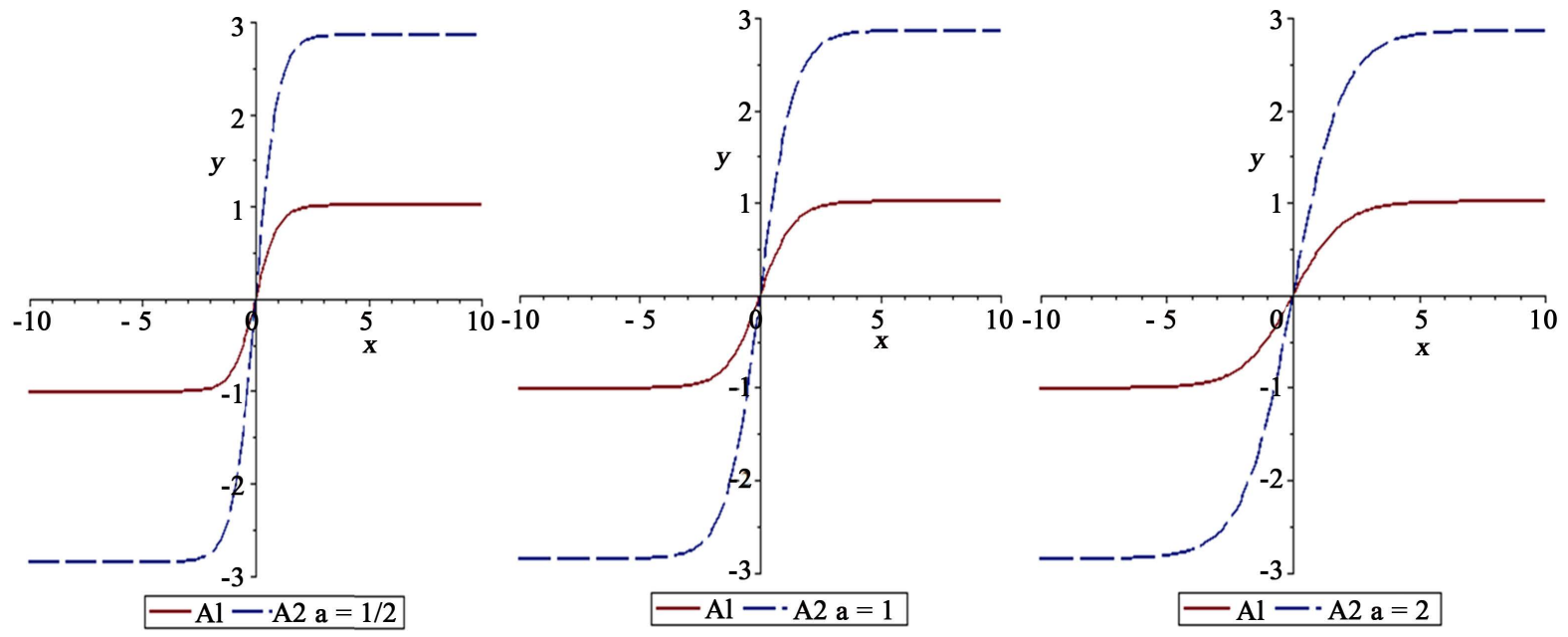

Figure 5. Dark solitons.
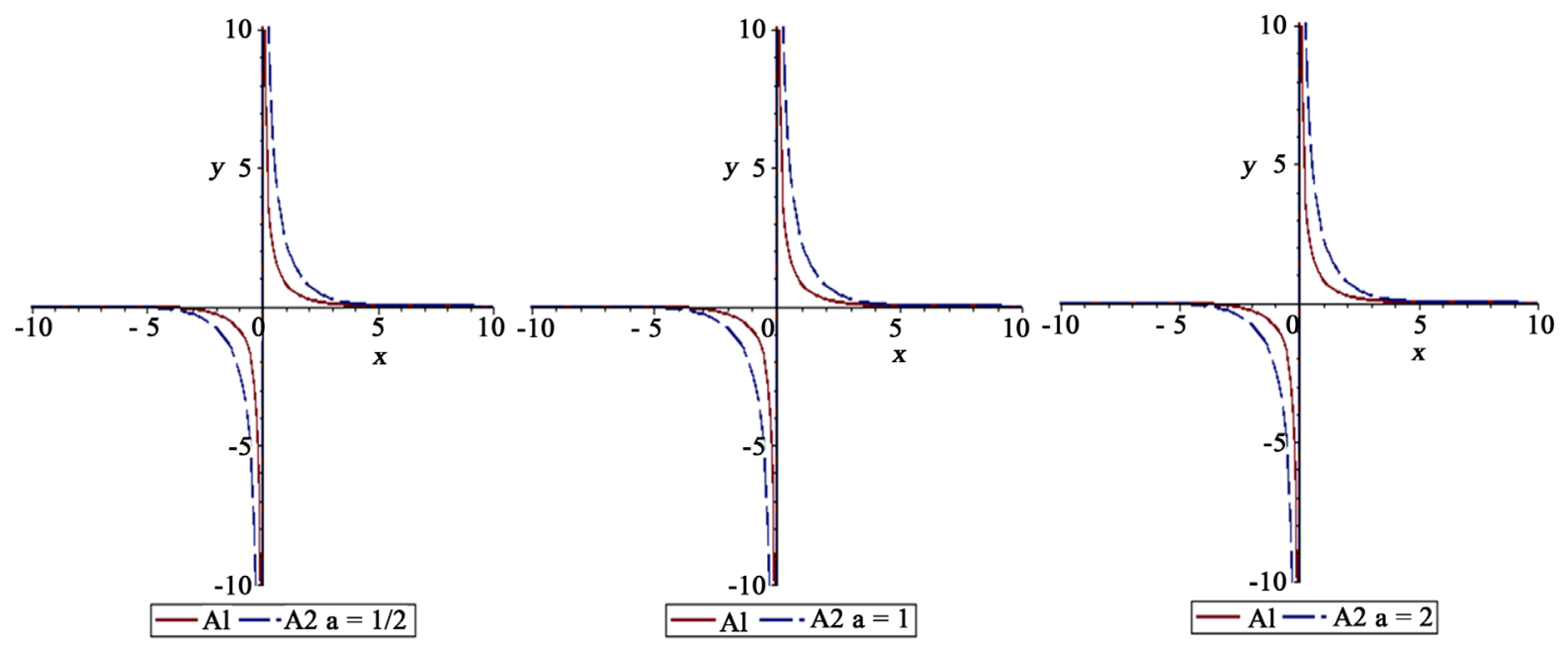

Figure 6. Singular solitons. 

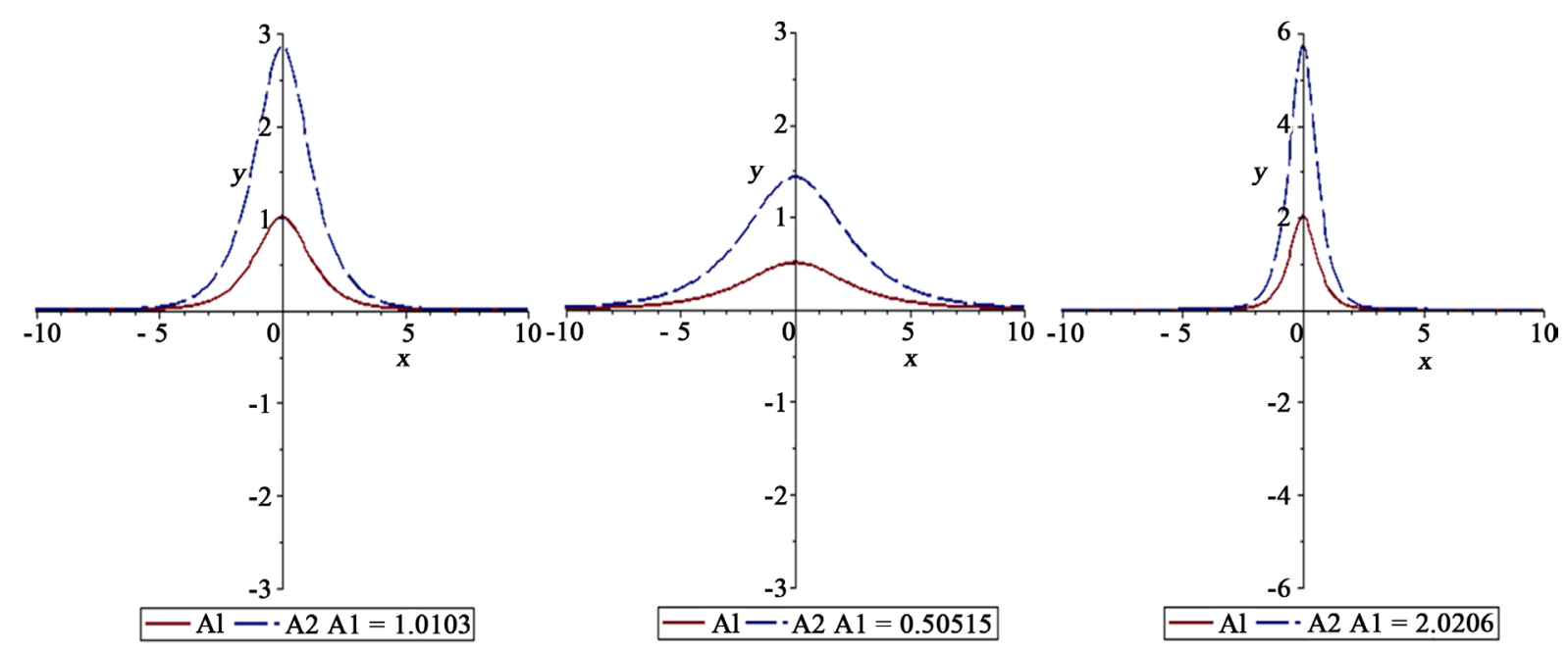

Figure 7. Bright solitons.

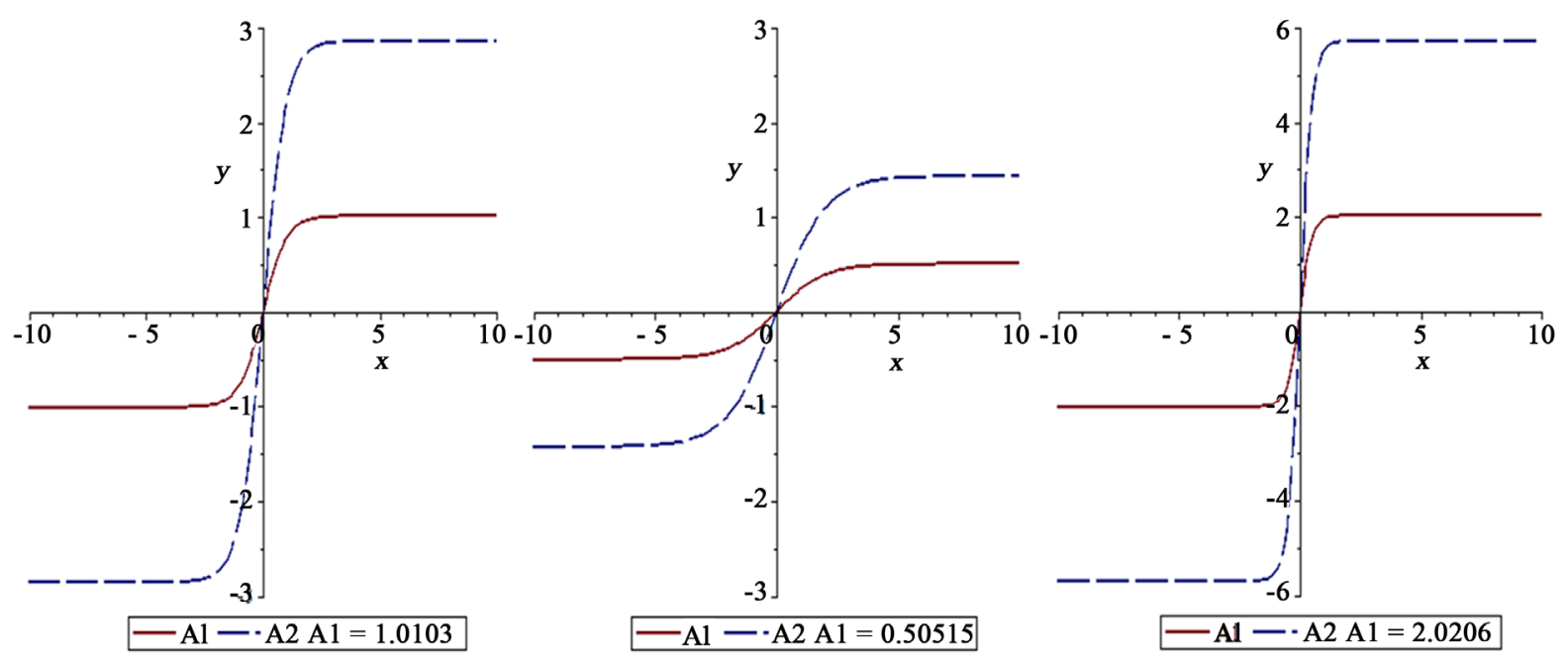

Figure 8. Dark solitons.
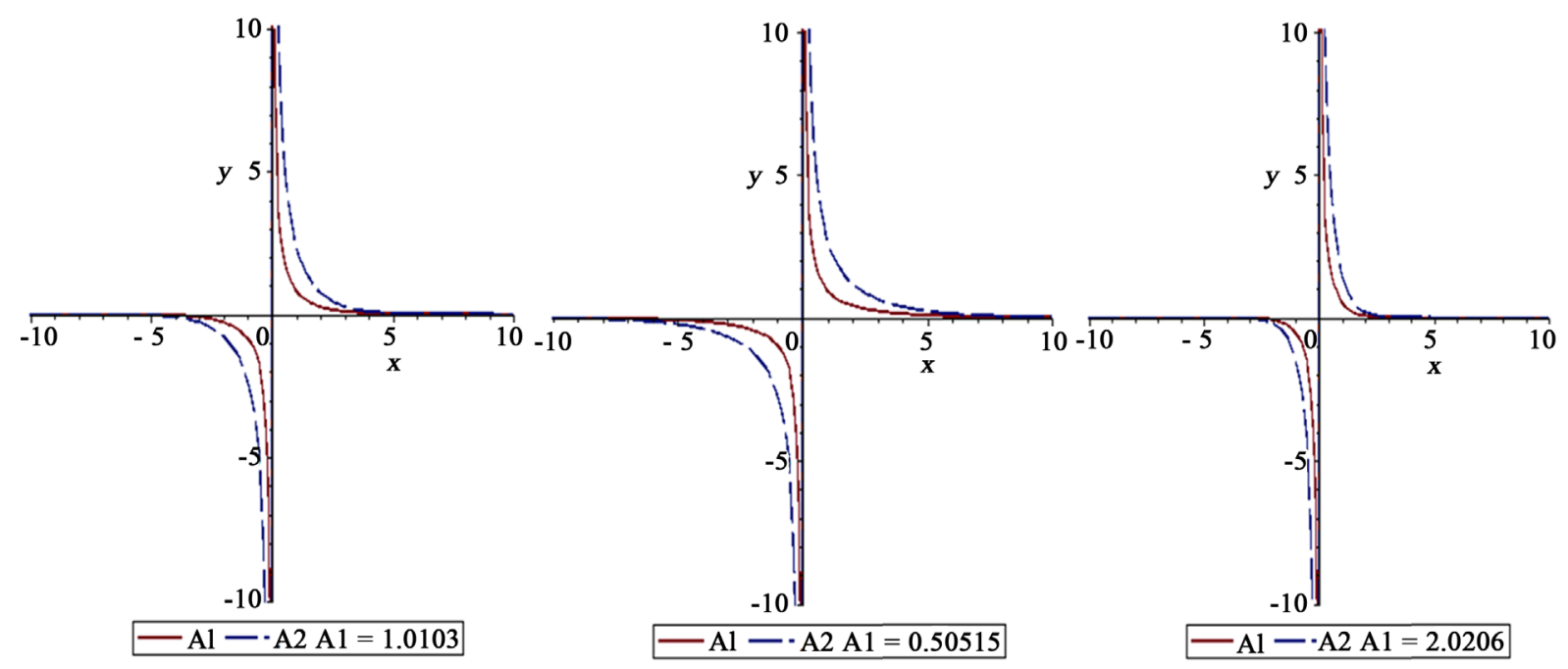

Figure 9. Singular solitons. 
offset the Schrodinger non-linear. As that finding, those solutions to some numerical methods provide a lot of add-ons in this study, and this is what we hope implemented with God's help in the future through the Master thesis.

\section{References}

[1] Biswas, A. (2003) Theory of Optical Couplers. Optical and Quantum Electronics, 35, 221-235. http://dx.doi.org/10.1023/A:1022852801087

[2] Zakharov, V.E. and Shabat, A.B. (1972) Exact Theory of Two-Dimensional Self-Focusing and One-Dimensional SelfModulation of Waves in Non-Linear Media. Journal of Experimental and Theoretical Physics, 34, 62.

[3] Ablowitz, M.J. and Segur, H. (1981) Solitons and H. Segur, Solitons and the Inverse Scattering Transform. SIAM, Philadelphia.

[4] Mihalache, D. and Panoiu, N.C. (1992) Exact Solutions of Nonlinear Schrodinger Equation for Positive Group Velocity Dispersion. Journal of Mathematical Physics, 33, 2323. http://dx.doi.org/10.1063/1.529603

[5] Mihalache, D. and Panoiu, N.C. (1993) Analytic Method for Solving the Nonlinear Schrodinger Equation Describing Pulse Propagation in Dispersive Optic Fibers. Journal of Physics, A26, 2679. http://dx.doi.org/10.1088/0305-4470/26/11/016

[6] Ramos, P.M. and Pavia, C.R. (2000) Self-Routing Switching of Soliton-Like Pulses in Multiple-Core Nonlinear Fiber Arrays. Journal of the Optical Society of America B, 17, 115.

[7] Biswas, A. (2001) Solitons in Nonlinear Fiber Arrays. Journal of Electromagnetic Waves and Applications, $15,1189$. http://dx.doi.org/10.1163/156939301X01101

[8] Biswas, A., Khen, K.R., Rahman, A., Yildirim, A., Hayat, T. and Aldossary, O.M. (2012) Journal of Optoelectronics and Advanced Materials, 14, 571.

[9] Sarescu, M., Bhrawy, A.H., Alsaery, A.A., Hilal, E.M., Khan, K.R., Mahmood, M.F. and Biswas, A. (2014) Optical Solitons in Nonlinear Directional Couplers with Spation-Temporal Dispersion. Journal of Modern Optics, 61, 441-458. http://dx.doi.org/10.1080/09500340.2014.894149

[10] Banaja, M.A., Alkhateeb, S.A., Alshaery, A.A., Hilal, E.M., Bhrawy, A.H., Moraru, L. and Biswas, A. (2014) Optical Solitons in Dual-Core Couplers. Wulfenia Journal, 21, 366-380.

[11] Biswas, A., Lott, D.A., Sutton, B., Khan, K.R. and Mahmood, M.F. (2013) Optical Gaussons in Nonlinear Directional Couplers. Journal of Electromagnetic Waves and Applications, 27, 1976-1985. 